research company, and included responses from 1,320 US adults aged 18 years or older.

The survey asked consumers a series of questions about health care topics, including, for example, who they most often see for their health care, who they would prefer to see if given the choice and what characteristics they would ascribe to various health care professionals.

The survey defined the term "medical team" and supplied a list of various health care professionals: nurse practitioners (NPs), physician assistants (PAs), internists, pediatricians, chiropractors, surgeons, physicians, and primary care physicians.

Respondents then were asked which professional on that list was their "first call" when a medical question arose, and $41 \%$ indicated that they wanted to confer with their primary care physician. Some of the other responses were physician (24\%), specialist (8\%), NP $(6 \%)$, and PA (3\%).

In addition, $70 \%$ of the adults surveyed said they were less likely to vote for a state representative who supported legislation that would restrict their ability to continue seeing their physician, $72 \%$ indicated they would be more likely to vote for a representative who would protect consumers' ability to continue to see their physician.

Blackwelder summarized the findings this way: "Finally, we have a survey from patients that says they not only value primary care, they value you for your education, expertise and experience.

"As family physicians, we not only are a critical part of the health care team, we are the identified leader of the team, and that's what our patients want and expect."

AAFP News Now

From the American

Board of Family Medicine at the University of Texas Health Science Center at San Antonio. He is a bicultural researcher and research ambassador to Latin America who advises US federal agencies developing health prevention projects for US Hispanic communities. Dr. Jaén also led the evaluation of the first national Patient-centered Medical Home demonstration. He continues to practice outpatient and inpatient medicine.

The Institute of Medicine (IOM) is unique in its structure as both an honorific membership organization and an advisory organization. Established in 1970 by the National Academy of Sciences, IOM has become recognized as a national resource for independent, scientifically informed analysis and recommendations on health issues. Fewer than 2,000 physicians are elected members, and just 70 US physicians are invited to join each year. The National Academy of Sciences was created by President Lincoln in 1863 .

Robert Pbillips, MD, MSPH, American Board of Family Medicine

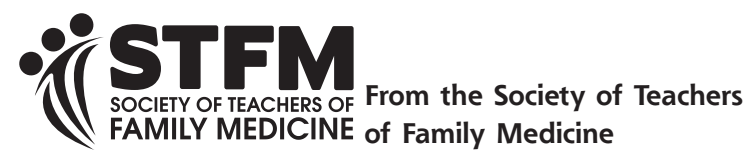

Ann Fam Med 2014;179-180. doi: 10.1370/afm.1632.

\section{STFM DEVELOPS PRECEPTOR GUIDELINES AND A POSITION STATEMENT ON STUDENT USE OF ELECTRONIC HEALTH RECORDS}

The Society of Teachers of Family Medicine has created 2 documents to help preceptors and other educators when working with students using electronic health records (EHRs). The guidelines will help preceptors work effectively with students when using EHRs. The position statement will assist preceptors and other educators when engaging compliance officers or organizations in discussions about the need for medical students to work meaningfully with electronic medical records.

\section{Preceptor Guidelines}

Medical students need to learn effective use of EHRs to prepare them for their work as residents and practicing physicians. This document reviews core medical student documentation issues and highlights core EHR concepts, functions, and skills to which we believe all medical students should have exposure and in which they should begin developing competence. As clinical preceptors we believe that actively considering these concepts and exposing your students to these concepts 
will enhance student learning and their ability to contribute meaningfully to the patient care experience. Read the Preceptor Guidelines Document at http:// www.stfm.org/resources/EHR.

\section{Position Statement}

CMS standards have significantly altered the involvement of medical students in the care of not only Medicare patients, but of all patients, as institutions, fearful of litigation, have enacted very conservative interpretations of the CMS standards. In some institutions, students are forbidden any access to EHRs; in other institutions the enacted policies have marginalized the role of medical students in the care of all patients. These CMS standards have also hindered the team function of care. The efficiency gained by the student's aid in documenting care has in the past provided time for the teaching physician to provide clinical instruction. CMS standards have largely eliminated this efficiency. Read the Position Statement at http://www.stfm.org/resources/EHR.

The documents are the work of the STFM Education Committee: Beat Steiner, MD, MPH, Chair Susan Cochella, $M D, M P H_{i}$ Bonnie Jortberg, $P b D_{i}$ Katie Margo, $M D_{i}$ Cbristine Jerpbak, $M D_{\text {i }}$ Allen Last, MD, MPH, Michael Mendoza, MD, MPH, Barbara Tobias, $M D_{i}$ Esther Jobnston, $M D, M P H_{i}$ Melissa Robinson

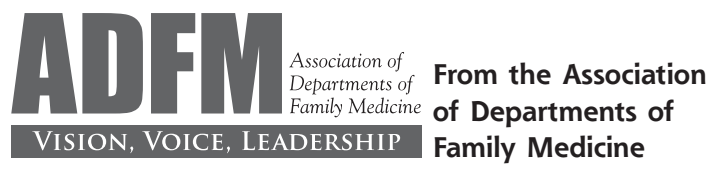

Ann Fam Med 2014;180-181. doi: 10.1370/afm.1628.

\section{HEALTH SYSTEM CHANGE AND ACADEMIC DEPARTMENTS}

Health system change is a now a reality in the United States and presents opportunities for family medicine to contribute rational solutions to the twin problems of high cost and variable quality confronting American health care. Academic health centers in particular will need to change if they are to survive and continue to make vital contributions to patient care, discovery, and educating tomorrow's health care workforce. Achieving the triple aim of improving the processes of care, improving the health of populations, and containing costs presents unique challenges to the siloed environments of academic centers. ${ }^{1}$ Can family medicine academic departments lead the way in health system change, and if so how?
The theme of the 2013 Association of Departments of Family Medicine (ADFM) Annual Winter Meeting was "Leading in the Time of Transition from Volume-Based to Value-Based Health Care." As part of this meeting, ADFM members engaged in workgroup discussions around 4 major themes: creating a strong partnership with the academic medical center (AMC); improving quality and reducing costs for patients, employers, and payers; collaborating effectively with other specialties; and improving the practice of family medicine and preparing the family physicians of the future. ${ }^{2}$ Following this session, a white paper with specific recommendations for how departments of family medicine (DFMs) can play a leading role in helping the nation with the transition to value-based health care was produced and shared with ADFM members. ${ }^{3}$

To ascertain how DFMs are meeting the challenge of health system change in their local environments - and the degree to which change has been implemented-questions about each of the specific recommendations were asked on the 2013 version of ADFM's annual member survey. Chairs of all 150 department members of ADFM, which include virtually all allopathic medical schools plus some osteopathic medical schools and large regional medical centers were invited to complete the survey. The survey included a number of other topics of interest to ADFM and DFMs and was sent electronically to department chair members using an online survey tool (Catalyst). The survey was open for 2 full months with regular reminders to those who did not complete the survey. The final response rate was $78 \%$.

Among the 117 respondents, 94\% were from allopathic medical schools; $74 \%$ from public institutions; and $47 \%$ from large schools (>149 students matriculating per year). A roughly equal number of respondents have been a department chair for more than 8 years $(39 \%)$ as those that have been a department chair for 3 years or less (38\%).

For the questions about the recommendations from among the 4 themes in health care transformation noted above, respondents were asked to "choose where your department is on each item" and were given the following options: not feasible in our setting; planned but not started ${ }_{i}$ piloting and/or in development imple- $_{i}$ mented-up and running.

Overall, DFMs across the country are playing a leading role in health care transformation. The changes that the most DFMs have currently implemented are: moving to team-based care in practices $(47 \%)_{i}$ improving delivery of preventive services $(42 \%)_{i}$ efforts to reduce avoidable hospital readmissions $(35 \%)$; making more appropriate use of consultations and referrals $(32 \%)$; and attracting and 